\title{
Improving access to competitive employment for service users in forensic psychiatric units
}

\author{
Charlotte Beck, Connie Wernham \\ John Howard Centre, East london NHS Foundation Trust
}

\begin{abstract}
Employment has been proven to be an effective recovery tool and therapeutic intervention for those with severe and enduring mental health conditions. Aside from monetary reward, employment is a means of structuring time and provides a sense of worth and achievement, which enhances self-esteem and confidence. A social identity is developed through employment, encouraging social support and increasing social networks. Securing employment can bring about improved quality of life and positive change in one's social circumstances; therefore it can reduce symptoms associated with mental illness and potentially prevent re-offending, as the individual develops a sense of independence, selfefficacy, and value.

Barriers to employment exist for forensic mental health service users and therefore it is imperative that employment needs are addressed at the earliest possible stage in recovery. An evaluation of employment activities across two forensic mental health units revealed a lack of appropriate employment opportunities for service users, and those roles available were not implemented in line with recommended best practice. In response to this issue several enterprises were established to offer opportunities for service users to engage in meaningful employment and develop skills that a future employer would value. Each enterprise responds to a business need within the units to ensure sustainability of services. The enterprises are essentially micro-businesses with social objectives whose surpluses are reinvested for the purpose of increasing opportunities for service users.
\end{abstract}

The enterprises are underpinned by the philosophy of the Individual Placement and Support (IPS) model; empirical evidence suggests that the IPS model is the most effective intervention, based on the 'place then train' philosophy. The model recommends a focus upon rapid job search to achieve competitive employment for those who want to work; opportunities sourced should be consistent with individual preference and benefits counselling offered. Support should be time unlimited and integrated with mental health treatment. A person-centred and strengthsbased approach is also adopted to support people to build on their strengths, establish goals, and encourage motivation.

\section{Problem}

The John Howard Centre and Wolfson House provide medium and low secure care within the East London area. The units provide therapeutic programmes for males and females diagnosed with a mental health condition and who are often considered to be a significant risk. Both units provide individualised treatment to meet the needs of a diverse client group. For example, services may be tailored for those with a learning disability or personality disorder.

Employment has been proven to be an effective recovery tool and therapeutic intervention (1). Evidence demonstrates that employment can reduce symptoms associated with psychiatric disorders, providing a route out of isolation, poverty and social exclusion, instilling hope, and increasing self-esteem and selfefficacy. Employment offers social rewards, providing opportunities to contribute to society and build social networks. Employment also moves people off benefits and decreases reliance upon NHS services (2-4). "A job provides money, social networks, relationships, confidence, satisfaction, personal fulfilment and a sense of achievement. This is what I am, and this is what I do. I am no longer a mental health condition" (5).

Securing employment can bring about improved quality of life and positive change in one's social circumstances. Therefore it can potentially prevent re-offending, as the emerging sense of independence, worth and value often acts as a protective factor to recidivism (6). Although there is some promising research in this area, evidence is inconclusive and further investigations are necessary. In particular, there is limited research into employment interventions targeted at offenders with mental health needs, and therefore insufficient evidence to determine conclusively the impact on re-offending.

While employment offers real benefits, accessing employment is often challenging for individuals diagnosed with a mental health condition. Additional hurdles exist for those with both a mental health condition and a history of offending behaviour. Barriers to employment may include the stigma associated with a mental health condition or offending history, employer perceptions, a fragmented employment history, out of date skills, a lack of confidence, low self-esteem, or an impairment affecting the scope of jobs available to an individual. These barriers will only increase during admission to a secure unit. Consequently, recovery through work is minimised and service users remain isolated and more likely to re-offend.

Though employment has been proven to be an effective recovery 
tool, employment opportunities are scarce within secure mental health settings. Employment type activities offered typically engage patients in the day to day running of their hospital environment, such as work in the laundry or cleaning, which have been developed to distract and calm individuals rather than improve their employability (7). Lack of early intervention increases barriers to employment, because being treated for long periods as a passive patient will often result in an inability to obtain and retain employment. Those services that are available post discharge are not often targeted at addressing needs associated with forensic mental health. Typically, employment services target either mental health or an offending history. Without employment those discharged from forensic mental health units have an increased risk of relapse and are often more likely to re-offend.

\section{Background}

There are two mainstream models of vocational rehabilitation; 'train then place' and 'place then train'. The 'train then place' approach focuses on structured activity and pre-vocational training to prepare service users for employment. This practice is dominated by concerns that the demands and stresses of employment will cause relapse. The 'place then train' model is based on the assumption that service users can work in competitive settings and therefore places them in employment, providing training while on the job. This method overcomes the job readiness obstruction where people become stuck in permanent training. It also increases motivation significantly since service users are employed from the beginning.

Empirical evidence suggests that the Individual Placement and Support (IPS) model is the most effective intervention, based on the 'place then train' philosophy (8-11). The key elements of the IPS model are as follows:

1. The focus is upon competitive employment

2. Support is integrated with mental health treatment

3. Support is open to those who want to work

4. Opportunities sourced are consistent with individual preference

5. Benefits counselling is provided

6. Rapid job searches are undertaken for either paid or unpaid work

7. Support is time unlimited and individualised.

Burns et al (1) compared train-place approaches with the IPS model. The authors documented significant increases in employment outcomes for those receiving employment support based on the IPS model ( $55 \%$ gained employment compared to $28 \%$ for other vocational services). Furthermore, there is no evidence to suggest that IPS increases the likelihood of clinical deterioration or relapse $(1,7,9,12,13,14)$. IPS participants sustained employment for longer and earned more. Unfortunately there is no equivalent research evidence available to assess the impact of IPS approaches to working with people with a history of offending behaviour.

\section{Baseline measurement}

In 2011, before project implementation, a review was undertaken to measure client needs regarding employment and to ascertain the extent of employment activities at the John Howard Centre, a medium secure psychiatric site. The employment review was conducted collaboratively, drawing on multidisciplinary expertise. Both quantitative and qualitative data were collected.

The qualitative research incorporated both surveys and focus groups for service users or staff. Both clients and staff agreed that employment was an important therapeutic intervention, and considered employment as a priority and central to recovery. There was also an emphasis on the importance of meaningful employment and a large amount of discussion centred around this and the definition. Clients' evidence of skill development was considered crucial to demonstrate progression and capability to an employer.

Findings suggested that there were no specific employment pathways in place to support service users into work. There were 131 jobs for service users on site, created to provide service users with employment rather than to fulfil a business need.

Consequently, there was little emphasis placed on job performance and no scope for progression or increased responsibilities within the roles. The jobs were non-standardised and managed individually by various teams and wards. As a result there was no consistency among the roles or monitoring to ensure hours and payments did not affect government benefits. The lack of central management drained both staff and monetary resources due to inefficiency. Finally, practices were not underpinned by research.

\section{Design}

Following the employment review it was recommended that employment provision should be underpinned with the principles of the IPS model and the occupational therapy paradigm, which recognises a person's need for occupational balance, and opportunities to maintain existing skills. Since February 2012, NuLeaf Enterprises has been developed as a sustainable vehicle for delivering the employment pathway. In 2014 Nu-Leaf Enterprises incorporates six projects, a café, two shops and a salon, as well as landscaping, picture framing and graphic design services (15). The enterprises offer opportunities for service users to engage in meaningful employment across a variety of sectors to develop skills that a future employer would value, thus significantly increasing their chances of securing employment in a competitive labour market. All paid jobs reflect community employment and the only criteria for referral is a motivation to work towards paid employment. The enterprises are businesses with social objectives whose surpluses are reinvested for the purpose of increasing opportunities for service users. Each of the enterprises responds to a real business need, for example, the landscaping service has won the contract to maintain the grounds for both the John Howard Centre and Wolfson House.

Each of the enterprises offers differing levels of risk, demands and responsibilities to meet personal interest and enable service users to engage at all stages of recovery. Referral to the project may be 
received from any of the wards in the John Howard Centre and Wolfson House, providing they have received approval from their clinical team. Service users are referred to the service via the occupational therapy team, who first evaluate the individual's skill set and complete a thorough risk assessment. Service users are initially offered an introductory session to find out more about the role, providing an opportunity to decide whether they would like to engage in unpaid work experience. If they wish to continue, work experience enables the development of a skill set in their chosen area. Upon completion of work experience, service users can then apply for paid employment as a team leader. The team leader's role is to support and train those on work experience, while taking responsibility for delivering service to a high standard. Service users undergo regular assessments throughout their engagement in the service to review personal development, areas for improvement, and goals to work towards.

Alongside the services, Nu-Leaf Enterprises delivers an employment course encouraging attendees to think about their journey into community employment. The course teaches attendees about disclosing a mental health condition or an offending history, permitted work, reasonable adjustments, how to apply for work, and how to interview effectively. Additional one-to-one support is provided for those who are nearing discharge to encourage engagement in community work experience and employment. IPS and strengths based approaches are adopted to support people to build on their strengths, establish goals, and encourage motivation.

\section{Strategy}

Initially the small scale enterprises were created to trial the employment model; this put emphasis on the benefits of training, industry-specific as well as transferable skills development, and generally equipping service users to increase their employability in a competitive employment market, despite the disadvantage of an adverse history of offending behaviour and mental health problems. Operationally, the existing three shops were condensed into two outlets, and a café was opened to provide an opportunity to develop a different skill set to the existing retail. Both shops and the café opened 2 days a week, due to staff resources, testing uptake of available unpaid work experience and practical assessment of risks involved.

As the work placements are unpaid and lack the incentive of instant gratification, one of the main concerns had been service user participation. What emerged was a change in the workforce from service users spending time in employment projects and getting paid regardless of performance, to intrinsically motivated individuals making pro-social choices and engaging with the service to minimise occupational disruption during their hospitalisation, maintain and develop skills, and increase their chances of paid employment post-discharge.

After only 8 weeks, the demand for work placements and access to the cafe demonstrated the success of the project and the need to expand. Based on the wealth of existing skills within the occupational therapy staff group who would facilitate further employment projects, as well as the desire to create a variety of work sectors to accommodate a range of opportunities, it was decided to establish a landscaping project, a picture framing project, and a design and print project. Service users also requested the establishment of a hair salon. All projects are now well established, and a number of service users work in multiple projects.

Individual progress is measured using a standardised occupational therapy assessment, the Work Star assessment, and recently a nonstandardised locally developed tool based on activity analysis. All assessments are carried out collaboratively, and Specific Measurable Achievable Realistic and Timely (SMART) goals are set to support and enable service users to achieve mastery of respective tasks, in order to be eligible to apply for the coveted team leader positions. Reviews of goals are scheduled on a 3 monthly cycle or to coincide with service user's 6 monthly Care Programme Approach (CPA) meetings, where their global progress is discussed and future care is planned by the multidisciplinary team with the service user, their carers, and legal representatives. However, informal assessments are ongoing in line with the Occupational Therapy Process, where function and performance are continuously monitored and goals and tasks are reviewed and adapted accordingly, in response to an increase or decrease in function and risk, or fluctuation of the employees' mental health. Once service users are comfortably established in a team leader role, settled within their treatment, and eligible for a suitable amount of leave, service users are encouraged to consider a placement at one of our community based partner organisations, to develop their skills and abilities in a community work environment.

\section{Results}

Service users are provided with real work experience, enabling valuable skills related to employment to be developed, such as punctuality, self-presentation, customer service, and accountability. By engaging in any one of the enterprises, service users enhance their CVs and gain an employer reference, supporting them to secure employment upon discharge. The service continues to produce successful outcomes.

Quantitative measures include the number of service users engaged in work tasters, work experience and employment (table 1), and improvements in health and wellbeing - measured using the Recovery Star and Work Star, number of hours spent facilitating services, and financial sustainability. Between January 2013 and January 2014 Nu-Leaf Enterprises' turnover has been approximately $£ 80,000$; all profits have been reinvested to develop services.

Qualitative measures include customer feedback, service user evaluation, and feedback from clinical teams, both directly and indirectly through clinical reports. The following statement has been provided by a service user involved in the landscaping enterprise: "Landscaping gave me a chance to do something positive, productive and improve my surroundings for myself and others... I learned about surveying and planning for the future. The work was interesting and changed with the seasons so new skills were being learned all the time. We got to know new people and build working 
relationships, which gave me energy and confidence to get into a job when I was discharged from the John Howard Centre... I learned a lot about horticulture and love how things grow and I use that principal in the Relationships I have and in my own recovery. Landscaping gave me a chance to stay in contact with the hospital, to keep trusted relationships going during my transition into the local community. This helps me stay on track on my road to recovery."

See supplementary file: ds3120.docx - "Number of service users engaged in the service January 2013 - January 2014"

\section{Lessons and limitations}

Forensic services are high risk environments where a careful balance must be maintained between security and therapeutic risk taking. The aim is to ensure that service users are cared for with the minimum restrictions and intrusions required to both meet their needs and to address issues of safety. It has therefore been challenging implementing enterprises in an environment where forensic service requirements are not necessarily beneficial for the business. For example, staff to patient ratios must be adhered to.

A further challenge was the requirement for Nu-Leaf Enterprises to meet running costs to ensure financial sustainability. Each of the enterprises has a regular turnover of team leaders and trainees, as individuals develop skills and progress into community employment. It has therefore been crucial that enterprises meet a real business need and yet create roles that allow for service users to train while in work to ensure continuity of services. Nu-Leaf Enterprises has been successful in reaching this goal.

\section{Conclusion}

Nu-Leaf Enterprises provides essential on-site services for a competitive price, while reducing patient stay, promoting recovery, and reducing the risk of re-offending. Nu-Leaf Enterprises has changed the culture within the John Howard Centre and Wolfson House, encouraging service users to have positive expectations of themselves and empowering them to believe that they can achieve something, and counteracting the stigma of the passive patient that sometimes prevails among healthcare professionals. The service user employment team encourages and supports the service users to make pro-social choices and provides them with a skill base and potential self-efficacy which will offer an alternative to re-offending in the long term. Engaging in employment provides an alternative route to assessing and reviewing the progress of service users and encourages the multidisciplinary teams to take a more global and long term view of the service user and their pathway.

\section{References}

1. Burns T, Catty J, Becker T, et al. The effectiveness of supported employment for people with severe mental illness: a randomised controlled trial. Lancet 2007; 370:1146-52

2. Waddell G, Burton A. Is work good for your health and wellbeing? London: The Stationery Office; 2006.

3. Ruesch P, Graf J, Meyer P, et al. Occupation, social support and quality of life in persons with schizophrenic or affective disorders. Soc Psychiatry Psychiatr Epidemiol 2004;39:686-94.

4. Burns T, Catty J, White S, et al. The impact of supported employment and working on clinical and social functioning. Schizophr Bull 2009;35:949-58.

5. Rickey B, Joy I, Paterson E. Job well done: employment and mental health problems, a guide for funders. New Philanthropy Capital; 2012, 1-25 .

6. Lipsey M. What do we learn from 400 research studies on the effectiveness of treatment with juvenile delinquents? In: McGuire J. What works? Reducing reoffending. New York: John Wiley; 1995, 63-78.

7. Rowland LA, Perkins R. You can't eat, drink or make love eight hours a day. The value of work in psychiatry. Health Trends 1988; 20:75-9.

8. Bond G. Supported employment: evidence for an evidencebased practice. Psychiatr Rehabil J 2004;27:345-59.

9. Bond G, Becker D, Drake R, et al. Implementing supported employment as an evidence-based practice. Psychiatr Serv 2001;52:313-22.

10. Bond G, Resnick S, Drake R, et al. Does competitive employment improve nonvocational outcomes for people with severe mental illness? J Consult Clin Psychol 2001;69:489-501.

11. Mueser K, Clark R, Haines M, et al. The Hartford study of supported employment for persons with severe mental illness. J Consult Clin Psychol 2004;72:479-90.

12. Lehman A. Vocational rehabilitation in schizophrenia. Schizophr Bull 1995;21:645-56.

13. Drake R, Becker D, Biesanz J, et al. Day treatment versus supported employment for persons with severe mental illness: a replication study. Psychiatr Serv 1996;47:1125-7.

14. Drake R, McHugo $G$, Bebout $R$, et al. A randomized clinical trial of supported employment for inner-city patients with severe mental disorders. Arch Gen Psychiatry 1999;56:627-33.

15. Nu-Leaf Enterprises [Internet] East London NHS Foundation Trust. http://www.eastlondon.nhs.uk/GetInvolved/NU-Leaf-Enterprises/NU-Leaf-Enterprises.aspx (updated 23 Nov 2013, cited Feb 20 2014).

\section{Declaration of interests}

Nothing to declare

\section{Acknowledgements}

